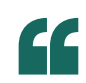

\section{We were \\ both surprised and excited \\ by the \\ advantageous \\ effect of \\ water}

\title{
Water promotes dehydration
}

In the design of new reactions, efforts are increasingly focused on the development of green processes - those that result in minimizing the use and production of hazardous materials. Of particular interest are the attempts to perform reactions in water or aqueous solvents. Now, writing in Nature Communications, Peizhong Xie, Teck-Peng Loh and colleagues report a greener method for the synthesis of allylic sulfones.

"We first became interested in organic reactions in aqueous media in the 1990s, when we found that indium complexes were useful and water-tolerant Lewis acids," explains Loh. "Later, we were working on the functionalization of proteins and found that the use of water-based reactions and metal-free conditions were necessary to preserve the tertiary protein structure. We now continue to further develop biocompatible water-based reactions."

Loh and co-workers were interested in synthesizing a series of trisubstituted allylic sulfones shown to be highly potent anticancer

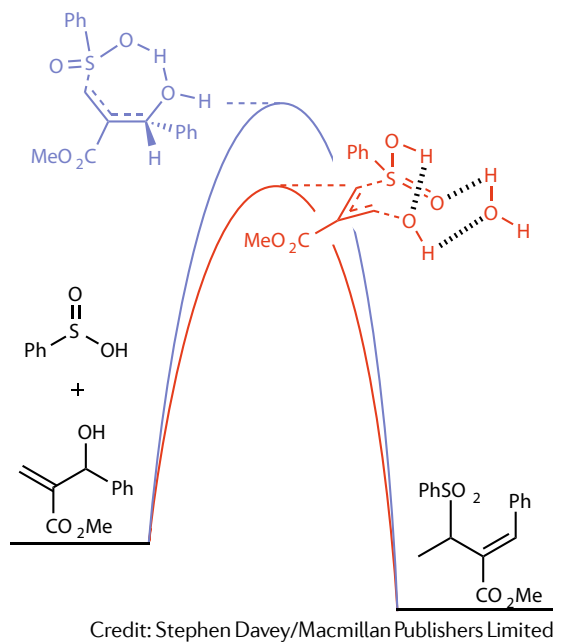

agents. It was thought that a coupling between an allylic alcohol and a benzenesulfinic acid would provide access, but initial reactions in toluene, while successful, gave poor yields. The team hypothesized that water may play an important role in the reaction. However, the reaction in water gave only $25 \%$ yield - this time attributed to poor reagent solubility - and so addition of a co-solvent was tested. Equal volumes of water and ethanol were found to provide the necessary balance affording the product in $75 \%$ yield by simple filtration.

Explaining the key advantages of this new reaction system, Loh said "This method works under biocompatible conditions (water/ethanol solvent system, room temperature, atmospheric pressure, $\mathrm{pH} 7$ ), and is atom economical with water as the only by-product. In most cases, the products precipitated directly from the reaction mixture, allowing for simple filtration and avoiding the need for organic extraction and chromatographic purificiation, enhancing the green credentials of the process."

In addition to being green, this new method shows excellent functional group tolerance, giving it the potential to be used in the late-stage allylation of complex sulfinic acids. Both electron-rich and electron-poor phenyl sulfinic acids were found to give excellent yields of over $90 \%$, and even reactions with alkylsulfinic acids, known to have lower reactivity, were successful.

Density functional theory calculations were carried out to help understand the importance of water. Hydrogen bonding was found to be the key factor, aiding the reaction in two ways. First, water mediates $\mathrm{H}$-bonding between the the allylic alcohol and benzenesulfinic acid - enhancing the nucleophilicity of the acid and electrophilicity of the alcohol - thereby accelerating the reaction process. Second, water was found to form a six-membered ring complex with the sulfinic acid. The reaction of this species with the allylic alcohol results in an energetically favoured transition state when compared with the reaction in the absence of water.

"We were both surprised and excited by the advantageous effect of water," says Loh. "The promotion of the reaction in water enables us to access our desired allylic sulfones under mild reaction conditions."

The research provides a metal-free, room temperature method for the synthesis of allylic sulfones, which can be carried out in aqueous media without the need for extraction or chromatography purification. Not only does the use of water work towards making this process more green but it actually acts to promote the reaction, giving improved conversion by the formation of a new, more favourable transition state.

Looking toward what the future might hold, Loh says "We believe that this study opens up the possibilities of designing new water-promoted organic reactions. We will be focusing on the application of this method for bioconjugation reactions, and the design of new water-promoted $\mathrm{C}-\mathrm{X}$ and $\mathrm{C}-\mathrm{C}$ bond formation reactions under biocompatible reaction conditions."

Katherine J. Geogheghan, Associate Editor, Nature Chemistry

ORIGINAL ARTICLE Xie, P. et al. Water-promoted C-S bond formation reactions. Nat. Commun. 9 , 1321 (2018) 